fact, it arises from a more important philosophical question-how geological time should be measured. When stratigraphy was in its infancy, it was natural to fix on spectacular events the effects of which were readily apparent and to use these for dividing the geological record.

More recent ideas on the philosophy of stratigraphy, however, suggest that time is a continuum, and that stratigraphic boundaries are merely arbitrary divisions, much as the hours of the day are arbitrary. Once a boundary is agreed, and defined by a type section, stratigraphers can get on with their real business-correlation. From this it follows that type sections should be chosen in areas where the rocks have all the features useful in correlation-good varied faunas (preferably marine) and as few obvious breaks as possible. This means that the traditional type sections of the Silurian/Devonian boundary in the Welsh Borderland just will not do-not only are half the rocks continental, but the famous Ludlow Bone Bed represents a marked time break. A very suitable type areat seems to bc the Barrandium Syncline near Prague, which was protected from the major movements of the Earth which disturbed north-west Europe in early Devonian times and where the graptolites, all-important in correlation. were not forced to extinction.

Geologists are agreed that the new proposal presents British stratigraphers with some difficulties, as Westoll and his colleagues point out. They suggest that the boundary should be fixed at another graptolite zone rather lower in the succession, marked by Monograptus ultimus, which would give a boundary very close to the position of the Ludlow Bone Bed.

Not all their British colleagues take this view; there is considerable British support for the new proposal. It will be interesting to see whether Westoll. Shirley, Dinely and Ball are fighting a rearguard action or starting a successful counter attack.

\section{OLFACTION}

\section{Smells Leff and Right}

from our Membrane Correspondent

IT has been reported that compounds, which differ only in their stereochemical configuration about one carbon atom, have distinguishably different odours. It is possible, however, that the difference in smell between these enantiomers can be attributed to that bugbear of olfactory research, the presence of unidentified impurities.

Earlier this year Leitereg et al. (Nature, 230, 455; 1971) presented good evidence that the optical isomers $(+)$ and (-)-carvones, prepared from both natural and synthetic sources, have different odours-caraway and spearmint respectively-but these authors still paid lip service to the possibility that the odour difference was caused by impurities. This possibility has now been convincingly eliminated in some recent work reported by Russell and Hills (Science, 172, 1043: 1971) and by Friedman and Miller (ibid., 1044).

Russell and Hills investigated five different enantiomeric pairs of compounds- $(+)$ - and $(-)$-carvones and four derivatives of each of these. The actual samples used for odour evaluation were purified fractions which were collected after successive passages through two different gas-liquid chromatography (GLC) columns. In each case there was a highly significant difference in odour quality. Using GLC and a variety of synthetic pathways, Friedman and Miller investigated eight different enantiomeric pairs which had distinct odour differences. All but one (amphetamine) of the pairs were terpenoid compounds and these included the carvones and also (+)- and (-)limonenes; the last two had the odour of oranges and lemons respectively-as in the case of the carvones this is a far from subtle difference in odour. Friedman and Miller paid particular attention to the carvones; they resolved a racemic mixture, synthesized them by two different routes and also subjected them to a cycle of reactions which interconverted the + form to the form and back again. The final products of all these manipulations were subjected to odour evaluation. The odours of the + forms were indistinguishable from each other as were the odours of the - forms, but the + and - pairs always had a significant difference in odour.

The postulated impurities now come to have unbelievable properties. There would have to be a different pair of impurities for many of the enantiomeric pairs studied, and in each case these impurities must survive both the GLC purification and the wide variety of chemical manipulations. Furthermore, just the same impurities would have to be present in both natural and synthetic compounds.

The vibrational and rotational modes of enantiomers are identical so that the now clearly established fact that enantiomers can have different odours spells death to any theory of olfaction which looks for correlations between odour quality and infrared spectra. Furthermore, other theories are now faced with the task of explaining why some enantiomeric pairs have different odours and other pairs have identical odours.

\title{
Some Pulsars May Emit Thermal X-rays
}

THE view that pulsars may consist of a superfluid core of neutrons surrounded by a rigid crust, as suggested by Baym, Pethick, Pines and Ruderman, is examined by George Greenstein of Princeton University Observatory in next Monday's Nature Physical Science. So far the attraction of the model of Baym and his colleagues has been that it accounts in a neat way for some of the irregularities in the behaviour of pulsars, interpreted in terms of starquakes occurring in the rigid crust. In this model the slowdown of the spin of the pulsar is accompanied by frictional heat as a result of slippage between the crust and the core.

Greenstein has evaluated the magnitude of this heating, which is on top of the temperature expected for pulsars on the basis of cooling theory, so that the temperatures which he obtains from an examination of the slowdown of pulsars can be considered as lower limits to pulsar temperatures.

It will not come as a surprise that the two pulsars for which the frictional heating process seems significant are the two fast pulsars, in the Crab Nebula and in Vela. For both these objects Greenstein finds temperatures caused by frictional heating arising between the rigid crust and neutron superfluid of $10^{6} \mathrm{~K}$. He suggests therefore that thermal X-ray emission ought to be detectable from these two objects---he expects them both to have X-ray fluxes of the order of $3 \times 10^{-27} \mathrm{erg} \mathrm{cm}^{-2}$ $\mathrm{s}^{-1} \mathrm{~Hz}^{-1}$.

It may also be possible to detect the thermal X-ray flux of $1 \times 10^{-28} \mathrm{erg} \mathrm{cm}^{-2}$ $\mathrm{s}^{-1} \mathrm{~Hz}^{-1}$ that is expected from what is thought to be the closest pulsar, CP 0950. Another possibility is that pulsars the orientation of which is such that their pulsations are not detectable at the Earth may be discovered by their thermal X-radiation.

But for other pulsars the expected thermal X-ray emission is appreciably weaker-1 $1 \times 10^{-30} \mathrm{erg} \mathrm{cm}^{-2} \mathrm{~s}^{-1} \mathrm{~Hz}^{-1}$ for HP 1508 and $3 \times 10^{-29} \mathrm{erg} \mathrm{cm}^{-2} \mathrm{~s}^{-1} \mathrm{~Hz}^{-1}$ for CP 1133. For these two objects the expected associated visual magnitudes are 32 and 27 respectively, far beyond the capabilities of the best optical telescopes.

Greenstein also reports that the heating at the inner boundary of the core takes place in a thin shell that can be a kilometre or so, or less, in thickness. $\mathrm{He}$ also points out that his equations indicate that fluctuations in the temperatures of pulsars are mirrored by corresponding fluctuations in the periodicity of the pulses, so that the regularity of pulsars implies that their temperatures are also kept amazingly constant. 